\title{
Revisiting the Reliability of the Endoscopy and Sedation- Assisted High-Resolution Esophageal Motility Assessment
}

\author{
Hassan Tariq, ${ }^{\mathrm{a}, \mathrm{b}}$, Jasbir Makker ${ }^{\mathrm{a}, \mathrm{b}}$, Chukwononso Chime ${ }^{\mathrm{a}, \mathrm{b}}$, Muhammad Umar Kamal ${ }^{\mathrm{a}, \mathrm{c}}$, \\ Ahmed Rafeeq ${ }^{a, b}$, Harish Patel ${ }^{a, b}$
}

\begin{abstract}
Background: Esophageal high-resolution manometry (HRM) is performed for evaluation of dysphagia or the pre-operative evaluation before esophageal surgery. The esophageal manometry parameters, interpreted as per the Chicago classification (CC), are meant to be acquired in an awake state. At times, the patient intolerance or inability to traverse the manometry catheter across the esophagogastric junction (EGJ) renders incomplete esophageal motility evaluation; hence, sedation or endoscopy assistance is required. There have been concerns raised regarding the use of sedation and resultant alteration of the manometry parameters. The aims were to study the effects of intravenous sedation on esophageal motility parameters and analyze its impact on outcomes of patients with dysphagia who are intolerant to awake manometry procedure.
\end{abstract}

Methods: The study population comprised patients who had sedation or the endoscopy assistance for the HRM. The indication for HRM, necessity for the sedation, manometry findings, barium esophagogram results, procedural timings and patient outcomes were reviewed. The diagnostic impact of the $10 \%$ correction in integrated relaxation pressure (IRP) was also studied.

Results: There were 14 patients from 179 awake manometry procedures that required the sedation or the endoscopy assistance. The mean age was 60.7 years and there was equal gender distribution. Dysphagia $(n=9)$ remained the predominant indication for the HRM, followed by the pre-operative evaluation for the esophageal surgery $(n=5)$. In eight patients, awake manometry failed due to the coiling of the catheter above the EGJ and six patients were intolerant to awake catheter insertion technique. Six patients were diagnosed with achalasia and two with EGJ obstruction. The correction of the possible $10 \%$ inflation of the IRP did not alter the final diagnosis in

Manuscript submitted April 19, 2019, accepted May 23, 2019

aDepartment of Medicine, BronxCare Health System, Bronx, NY 10457, USA bivision of Gastroenterology, Department of Medicine, BronxCare Health System, Bronx, NY 10457, USA

'Corresponding Author: Muhammad Umar Kamal, Department of Medicine, BronxCare Health System, Bronx, NY 10457, USA.

Email: muhammadumarkamal@gmail.com

doi: https://doi.org/10.14740/gr1185 majority except one patient with the EGJ obstruction. The findings of the barium esophagogram corroborated the manometry diagnosis.

Conclusion: Esophageal HRM should be done in awake state as much as possible. Sedation may be a feasible option as against aborting the further workup in patients who fail with current techniques involving awake catheter insertion. However, one needs to be mindful of sedation effects on manometry parameters and interpret results carefully.

Keywords: High-resolution manometry and sedation; HRM in intolerant patients; Esophageal manometry and anesthesia; Reliability of HRM and sedation; Failed awake HRM

\section{Introduction}

The recent advances proposed by the International High-Resolution Manometry (HRM) Working Group have led esophageal motility evaluation to a new horizon [1]. The impendencebased multi-channel HRM has been the standard of care for esophageal manometry assessment. The procedure involves trans-nasal esophageal catheter placement and recording the rhythmic contraction and sequential relaxation of the esophagus and esophagogastric junction (EGJ). The Clouse plot is obtained from the topographic pressure data of HRM [2]. The algorithmic scheme of Chicago classification (CC) criteria of esophageal motility disorders proposed the hierarchical analysis for the diagnosis of the motility disorders [3]. Basic measurements obtained during esophageal HRM as defined by CC include the integrated relaxation pressure (IRP), distal contractile integral (DCI), distal latency (DL) and the contractile front velocity (CFV). The hierarchical algorithm evaluates the IRP and subsequently the failed peristalsis, DL and DCI to identify the motility disorders [1]. Hence, it is imperative to acquire and interpret all the measurements as per the $\mathrm{CC}$ protocol.

The CC protocol recommends acquisition of the esophageal manometry with ten $5 \mathrm{~mL}$ saline swallows in the supine position. The standard of care is to perform conscious transnasal catheter insertion. The poor tolerance [4], inability to traverse the EGJ in patients with the altered gastroesophageal anatomy [5] and nasopharyngeal anatomy pose difficulty with the catheter insertion. The problem of poor tolerance to cath- 
eter insertion while awake can be circumvented by utilizing sedation. Direct vision-guided catheter placement across the EGJ can be achieved with an upper endoscope. Hence endoscopy-assisted motility catheter placement in a sedated patient can assist with HRM in patients with poor tolerance.

The measurements, as per the $\mathrm{CC}$, are to be acquired in a standard setting as defined by the patient position, consistency and quantity of the swallow. Any deviation from the protocol may alter the results. The changes in the position and consistency of the swallowed bolus have been shown to affect manometry findings $[6,7]$. Post-sedation status may impact the manometry measurements. Currently, there is a scarcity of the data exploring effects of sedation on manometric esophageal parameters. We opine that sedation-assisted esophageal manometry is frequently practiced but seldom reported $[8,9]$. Hence, to guide the management of such patients where sedation-assisted HRM is utilized, it is imperative to conduct more research exploring this group of patients. It is also essential to interpret esophageal manometry results in conjunction with clinical presentation before implementing changes in the management. We present our experience of HRM with sedation-assisted endoscopic placement of the manometry catheter. The impact of the sedation on HRM parameter, though not certain, has been discussed later in our study. We reviewed the prior literature to review the impact of sedation on the key clinical findings like IRP [10]. There have been prior animal studies that suggest the impact of sedation on the key esophageal motility findings [11]. Based on the prior literature, we implemented sedation-related correction to the key HRM finding of IRP, and evaluate if it alters the esophageal manometry diagnosis.

In our study, we do intent to describe the procedure and tips for the physicians planning to incorporate sedation assistance for the HRM catheter placement.

\section{Materials and Methods}

This is a retrospective review of esophageal manometry studies preformed at a single center between September 2010 and December 2018. The research protocol conforms to Declaration of Helsinki. The study protocol was reveiwed and approved by the Instituional Review Boad (IRB) at BronxCare Hospital Center.

\section{Study population}

Data for all the patients who underwent esophageal manometry during the study period were extracted. The patients who had the endoscopy assitance for the HRM were identified. The procedure docementation was reviewed to confirm utilization of endoscopy assistance for HRM. The age, gender, race and self-reported ethnicity were recorded.

\section{Clinical history}

The medical charts were reviewed and indiciations of the initial HRM were noted. The reasons for intolerance to awake HRM were also reviewed. The manometry studies performed under sedation with endoscopy for these patients who failed initial awake manometry were reviewed. The clinical outcomes and management after the diagnosis were also reviewed.

\section{Manometry findings}

All the patients who failed initial awake HRM eventually completed the exam under sedation with endoscopy guidance. The reprots of the HRM were reviewed. If the results of the study were reported using the prior conventional method, then the old study was retrieved, if avaiable, to re-analyze them as per the most recent CC. The results in this study are presented as per CC. The studies were analyzed utilizing the same software which was used to acquire the study, namely bioview analysis software program (Sandhill Scientific, inc, Highlands Ranch, $\mathrm{CO}$, USA) and Zvu ${ }^{\circledR}$ advanced G.I. diagnostic software (Diversatek, Milwaukee, WI, USA).

\section{Procedure description}

A $1.5 \mathrm{~h}$ procedure time slot was booked for each patient. The Insight Ultima ${ }^{\circledR}$ G3 manometry system (Diversatek Health Care) was used for the HRM. The manometry catheter caliberation was performed prior to patient arrival in the endoscopy room and the catheter was kept ready for insertion. Nurse and anesthesia staff were explained about the procedure and also instructed to avoid using long acting sedatives. The patients provided informed consent for both esophagogastroduodenoscopy (EGD) and esophageal HRM. In patients with intolerance to awake catheter insertion as an indication for the HRM, a second attempt to place the catheter was made under sedation prior to the endoscopy. To assist in awake catheter insertion, local anesthetic lidocaine and techniques like making patient sip water through a straw during catheter insertion were utilized. If cather placement was successful, then endscopy was not performed. When needed, the endoscopy was performed prior to the catheter insertion. During EGD, the scope was held at $30 \mathrm{~cm}$ from the insciors. The trans-nasal cather insertion was performed. If the catheter was not visualzed in the esophagus during insertion, then scope was withdrawn to oral cavity and catheter was manuevered through the oropharynx into the cervical esophagus under direct vision with the endoscope. The passage of the catheter across EGJ was photo documented (Fig. 1). The scope was withdrawn while providing the torque in alternating direction. This withdrawal technique avoids the single point contact and prevents the displacement of the manometry catheter.

After the scope withdrawal, the lower esophageal sphincter (LES) manometry tracings were reviewed to confirm the appropriate placement. Patient was turned to supine position and anesthesia staff was instructed to stop the sedation. The endoscopist remained at the bed side and monitored the capnograph to assess the sedation reversal. Patient required careful supervison to prevent them from pulling the catherter on regaining the conciousness.

The resting phase of the study was recorded while they were regaining the conciouness. The manometry acquiring protocol was paused after the acquistion of the resting phase. 


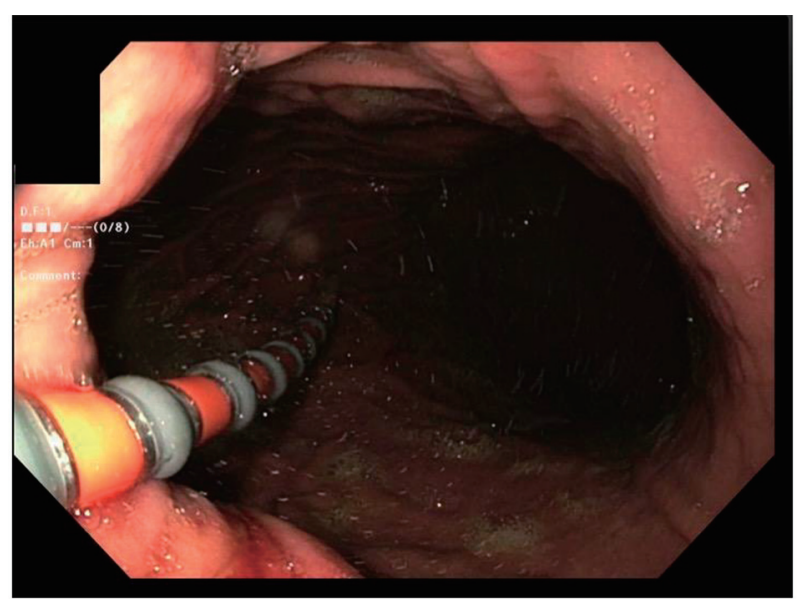

Figure 1. The passage of the catheter across esophagogastric junction (EGJ).

After patient was fully awake and following commands as assessed by anesthetist as well as endoscopist, the test swallows were given and subsequent parts of the study were completed. The HRM report was annotated for the use of the sedation.

\section{Procedural parameter}

The anethesia time tracking in the electronic medical record was used to document the duration of the procedure. The duration of the endscopy, recovery and the manometry were noted to calculate the total procedure duration. Significant events like hypoxia, hypotension or arrythmia, if any, were also re- viewed and recorded from the anesthesia notes. These findings are essential to evaluate for the safety of the procedure.

\section{Results}

A total of 179 esophageal HRM procedures were performed utilizing local anesthetic lidocaine with the patient in an awake state. All the procedures were performed by a gastroenterologist. Seven percent $(n=14)$ of procedures could not be completed and were aborted due to failure to pass manometry catheter beyond EGJ. All of these 14 patients underwent esophageal HRM catheter insertion under sedation. In two patients, the catheter could be negotiated with sedation only and 12 required the endoscopic assistance for catheter insertion. The mean age of study group was 60.7 years. The study group comprised $50 \%$ women $(n=7)$ and $50 \%$ men $(n=7)$. Indications for HRM included evaluation of dysphagia $(n=9)$, preoperative evaluation of hiatal hernia repair and fundoplication $(\mathrm{n}=3)$, fundoplication revision pre-operative evaluation $(\mathrm{n}=$ 1) and post-operative distal esophagectomy dysphagia evaluation $(n=1)$. Among these patients, indication for sedation or endoscopy assistance included inability to traverse EGJ and coiling of catheter $(n=8)$, patient discomfort and/or coiling of the catheter $(n=6)$. Details are presented in Table 1 .

The CC criteria of esophageal motility disorders (V3) was utilized for the esophageal manometry evaluation. Patients with elevated IRP with aperistalsis were diagnosed with type I achalasia; elevated IRP with isobaric pan-esophageal pressurization were diagnosed with type II achalasia; elevated IRP with simultaneous esophageal contractions were diagnosed with type III achalasia. Patients with elevated IRP and normal peristalsis

Table 1. Demographic Information, Indications for HRM and Endoscopy Assistance Among the Patients Included in the Study Group

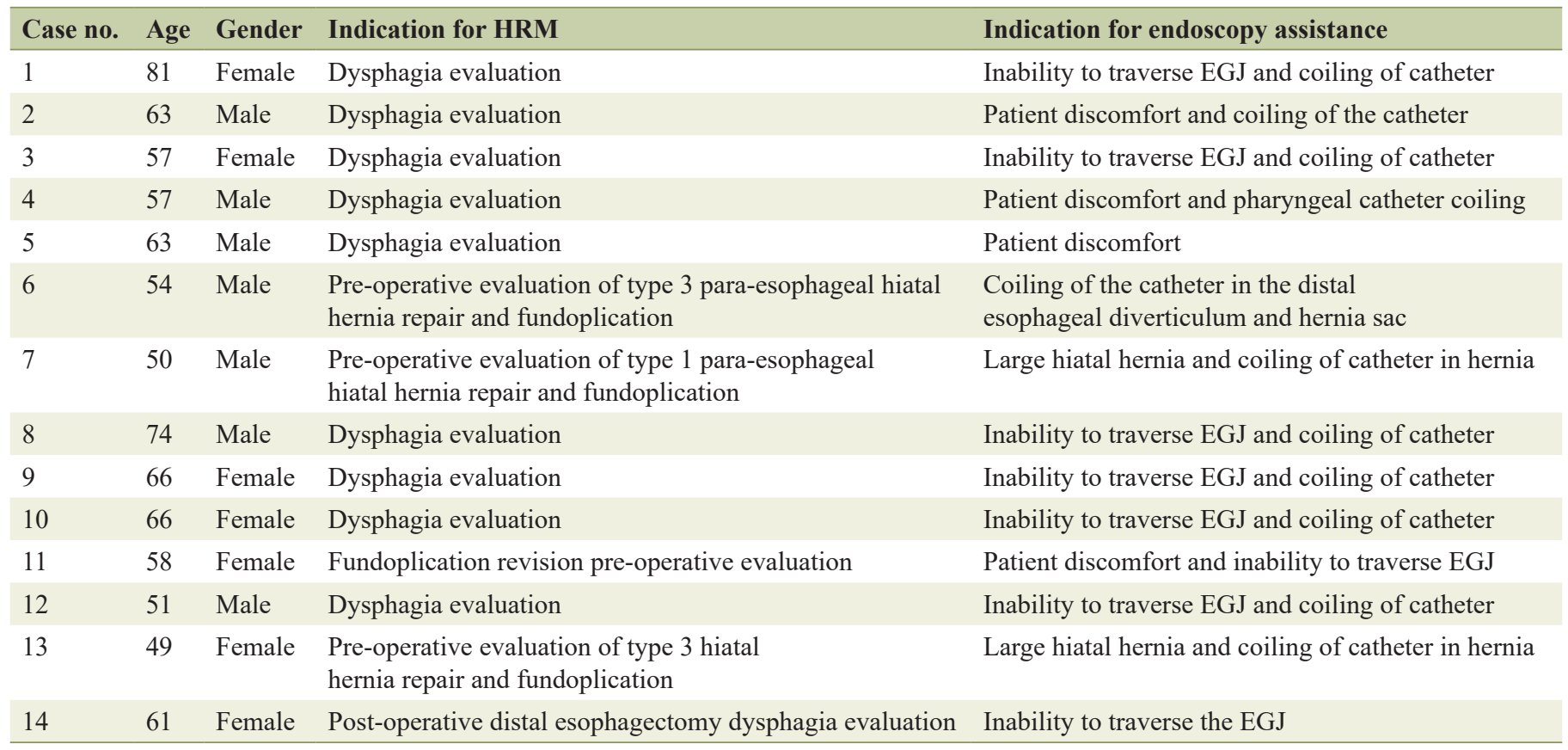

HRM: high-resolution manometry; EGJ: esophagogastric junction. 
Table 2. Findings on Manometry, Barium Esophagogram and Diagnosis of Patients in the Study Group

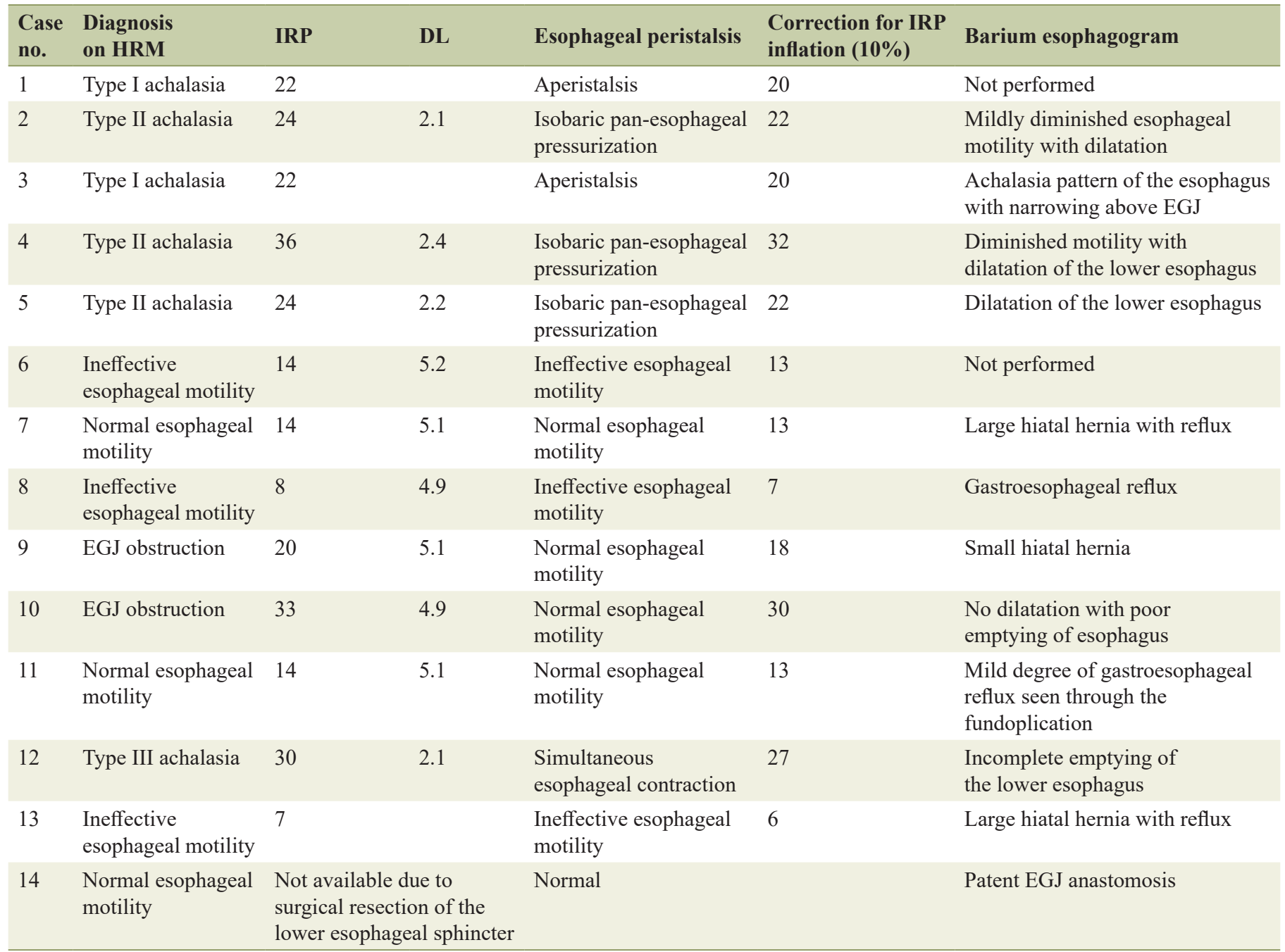

HRM: high-resolution manometry; EGJ: esophagogastric junction; IRP: integrated relaxation pressure; DL: distal latency.

were diagnosed with EGJ obstruction. The presence of normal IRP but ineffective peristalsis in $>50 \%$ swallows was diagnosed as ineffective esophageal motility. Most patients who underwent an esophagogram had an abnormality reported especially in patients who were later diagnosed as having achalasia (Table 2).

Among the 14 patients, six patients were diagnosed with achalasia (type $\mathrm{I}=2$, type $\mathrm{II}=3$, type III $=1$ ), three patients with ineffective esophageal motility, two with EGJ obstruction and three patients had normal motility. In the absence of studies exploring the sedation effect on key manometry parameters like IRP, it is not known how to interpret the esophageal manometry studies done under sedation. As discussed later, few studies have shown increase in lower esophageal residual pressure after use of midazolam or opiates. Lower esophageal residual pressure essentially means IRP; hence these studies with midazolam and opiates suggest increase in IRP. However, the effect of propofol on IRP has not been studied. The two studies which came close to study this effect analyzed the effect of propofol sedation on LES pressure but not the LES re- laxation which is the key factor determining IRP. Moreover, results from these two studies about effect on LES pressure are conflicting. If the results of these studies are to be extrapolated to interpret the effect of sedation on IRP, it remains undetermined whether IRP will increase or decrease after sedation. With the current scarcity of data on such an issue, we decided to see how the management will change if a $10 \%$ decrease in IRP is applied to the acquired IRP from manometry studies done under sedation. We did not apply $10 \%$ increase in IRP as that will anyway make the abnormal results more significant. The 10\% downward adjustment in IRP did not change the diagnosis in six patients with achalasia (cases 1-5 and 12) where diagnosis was also supported by the altered esophageal motility and the barium esophagogram or a computed tomography (CT) scan (Fig. 2). In four patients with ineffective esophageal motility (cases 6-8 and 13) and three patients with normal motility study (cases 7, 11 and 14), adjustment in IRP did not make any change in the final diagnosis. Out of two patients with EGJ obstruction (cases 9 and 10), one retained the diag- 


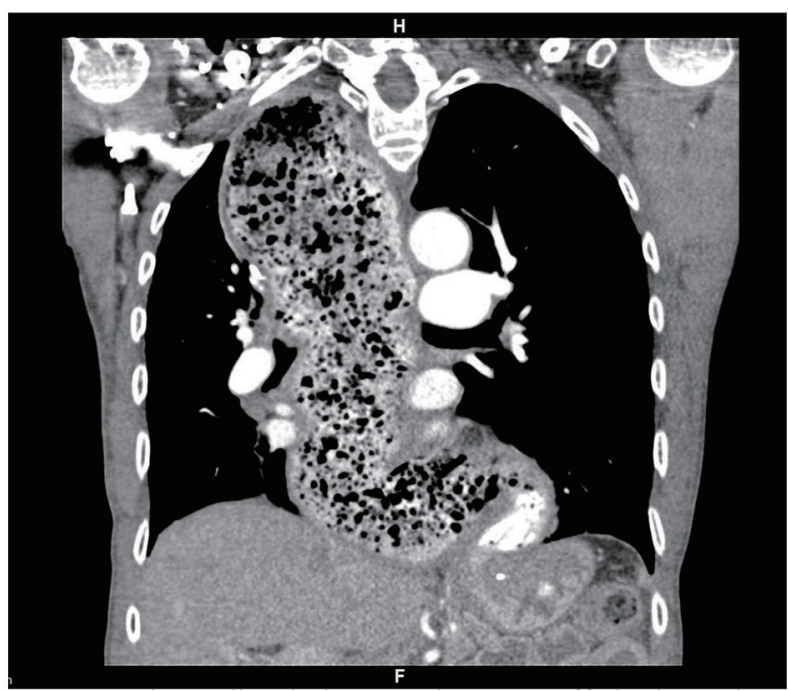

Figure 2. Computed tomography (CT) scan showing a dilatated and tortuous esophagus, presence of food residue, smooth-tapered appearance (bird beak sign) of the esophagogastric junction (EGJ) and loss of gastric air bubble characteristic of achalasia.

nosis (case 10) despite the IRP adjustment but the other patient (case 9) with pre-adjustment IRP 20 had a change in diagnosis, though it did not change the management.

\section{Management of study population}

\section{Patients with achalasia}

Both patients with type I achalasia refused Heller's myotomy. One of these patients underwent balloon dilation but the other patient refused balloon dilation as well, and hence was managed with calcium channel blockers. Among three patients with type II achalasia, two patients underwent Heller's myotomy, while one patient who refused Heller's myotomy was managed with calcium channel blockers. The patient with type III achalasia underwent Heller's myotomy.

\section{Patients with ineffective esophageal motility}

Among three patients with ineffective esophageal motility, one patient underwent Nissen's fundoplication, one patient is undergoing evaluation for Nissen's fundoplication and one patient was managed with acid suppression as patient refused surgery.

\section{EGJ obstruction}

Both patients with EGJ obstruction underwent observant management and will undergo follow-up imaging.

\section{Normal motility}

Among three patients with normal motility, one underwent Toupet's $270^{\circ}$ fundoplication as planned for management of GERD, one patient is planned for revision of fundoplication, while one patient who had dysphagia after distal esophagectomy underwent dilatation of the esophago-gastric anastomosis.

These findings are tabulated in Table 3.

\section{Interval from diagnosis to management}

Among the patients with type I achalasia, the pharmacological

Table 3. Diagnosis of Patients Undergoing Endoscopy-Assisted HRM and Their Management/Treatment

\begin{tabular}{lll}
\hline Case no. & Diagnosis on manometry & Treatment \\
\hline 1 & Type I achalasia & Patient refused balloon dilatation and Heller's myotomy - managed with calcium channel blocker \\
2 & Type II achalasia & Heller's myotomy \\
3 & Type I achalasia & Balloon dilation, patient refused Heller's myotomy \\
4 & Type II achalasia & Patient refused balloon dilatation and Heller's myotomy - managed with calcium channel blocker \\
5 & Type II achalasia & Heller's myotomy \\
6 & Ineffective esophageal motility & Nissen's fundoplication \\
7 & Normal esophageal motility & Toupet's $270^{\circ}$ fundoplication \\
8 & Ineffective esophageal motility & GERD management \\
9 & EGJ obstruction & Observant management \\
10 & EGJ obstruction & Observant management \\
11 & Normal esophageal motility & Planned for the revision of the fundoplication \\
12 & Type III achalasia & Heller's myotomy \\
13 & Ineffective esophageal motility & Undergoing evaluation for Nissen's fundoplication \\
14 & Normal esophageal motility & Dilatation of the esophago-gastric anastomosis \\
\hline
\end{tabular}

HRM: high-resolution manometry; EGJ: esophagogastric junction; GERD: gastroesophageal reflux disease. 
Table 4. Duration of Manometry Catheter Calibration, Endoscopy, Post-Sedation Recovery, Manometry Acquisition and Total Procedure Duration

\begin{tabular}{llllll} 
Case no. & $\begin{array}{l}\text { HRM catheter } \\
\text { calibration (min) }\end{array}$ & EGD duration (min) & $\begin{array}{l}\text { Post-sedation } \\
\text { recovery (min) }\end{array}$ & $\begin{array}{l}\text { HRM acquisi- } \\
\text { tion (min) }\end{array}$ & $\begin{array}{l}\text { Total procedure } \\
\text { duration (min) }\end{array}$ \\
\hline 1 & 12 & 16 & 32 & 22 & 82 \\
2 & 10 & 7 & 24 & 18 & 59 \\
3 & 13 & 8 & 31 & 25 & 77 \\
4 & 14 & 12 & 22 & 19 & 67 \\
5 & 12 & 11 & 36 & 21 & 80 \\
6 & 10 & 9 & 26 & 20 & 65 \\
7 & 12 & 16 & 36 & 22 & 86 \\
8 & 16 & 11 & 24 & 21 & 72 \\
9 & 12 & 10 & 32 & 20 & 74 \\
10 & 10 & 11 & 28 & 18 & 67 \\
11 & 14 & 18 & 36 & 18 & 86 \\
12 & 12 & $2 *$ & 24 & 16 & 54 \\
13 & 10 & 16 & 22 & 18 & 66 \\
\hline
\end{tabular}

${ }^{*}$ Catheter insertion with the sedation and no endoscopy. HRM: high-resolution manometry; EGD: esophagogastroduodenoscopy.

intervention with the calcium channel blocker was initiated in 1 week after the diagnosis and the other patient underwent the balloon dilatation 6 weeks after the diagnosis. Two patients with the type II achalasia underwent Heller's myotomy at 14 and 16 weeks respectively and the third individual was initiated on the pharmacology intervention at 18 weeks. The patient with type III achalasia had Heller's myotomy 20 weeks after diagnosis. Patient declined the referral for per-oral endoscopic myotomy (POEM). The patient with the EGJ obstruction had observant management. Patients with the pre-fundoplication motility assessment were awaiting the procedures. There was one patient with the distal esophagectomy who underwent dilatation of the esophago-gastric anastomosis 2 weeks after the motility study.

On an average, the entire procedure required $70 \mathrm{~min}$ with majority of the time $(28 \mathrm{~min})$ spent in post-sedation recovery. The EGD required around $12 \mathrm{~min}$ and HRM acquisition required $20 \mathrm{~min}$. The details are presented in Table 4 . These findings are presented for the time estimation and endoscopy slot planning.

All patients received intravenous sedation with propofol, while 13 of 14 patients received lidocaine as well. At discretion of the anesthesiologist, some patients also received metoprolol, fentanyl and midazolam (Table 5). In two patients that received fentanyl, we re-evaluated manometry findings and found no abnormalities.

\section{Discussion}

The pre-procedural assessment plays a key role in the postprocedure interpretation. Physicians should have a thorough insight in need for sedation and endoscopy for the HRM. The suggested indications for sedation use, as in our study, are nasopharyngeal intolerance, excessive pharyngeal gagging, lower esophageal coiling and inability to pass through the EGJ or LES. The catheter placement can be attempted with minimal sedation in the patient with the nasopharyngeal intolerance or gagging. The lower doses of propofol have been shown to minimally affect the LES in young individuals [12]. This initial attempt with minimal sedation, if successful, may mitigate sedation effect on key motility findings. Subsequently, endoscopy-assisted catheter placement can be performed if the initial attempts with minimal sedation are unsuccessful. Patients with moderate to large hiatal hernia or prior lower esophageal surgery, where chances of manometry catheter getting coiled in lower esophagus are higher, can proceed with endoscopic assistance as the initial step.

One should have a clear understanding of the hierarchal steps involved in assessing an esophageal motility disorder. The evaluation of the LES obstructive pathology remains the key initial step in the overall evaluation [1]. Turan et al reported the effect of the propofol on LES pressure. The LES pressure increases at the lower dose with target site concentration of propofol of $1 \mu \mathrm{g} / \mathrm{dL}$; however, it does lower the LES pressure at moderate sedation doses [13]. Their study intended to review the effect of sedation on LES tone and imply the outcomes towards the gastric content aspiration during the anesthesia. The manometry was performed with the four-sensor manometry catheter with the identification of the LES with the pull through technique [14]. This certainly has limitation in implementing the results from this study to now prevalent technology of the HRM.

Leon et al, while studying clinical implication of sedation on esophageal manometry [12], reported findings that were different from Turan et al. Their study revealed that the ef- 
Table 5. Medications Used for Anesthesia Among Patients in the Study Population

\begin{tabular}{|c|c|c|c|c|c|}
\hline Case no. & Propofol (mg) & Lidocaine (mg) & Metoprolol (mg) & Fentanyl ( $\mu \mathrm{g})$ & Midazolam (mg) \\
\hline 1 & 50 & 50 & & & \\
\hline 2 & 100 & 50 & 3 & & \\
\hline 4 & 70 & 50 & 3 & & \\
\hline 5 & 90 & 50 & 3 & & \\
\hline 8 & 80 & 50 & & & 3 \\
\hline 9 & 70 & 50 & & & \\
\hline 10 & 60 & 50 & 3 & & \\
\hline 11 & 120 & 50 & 3 & 100 & \\
\hline
\end{tabular}

fect of propofol on LES was more pronounced in the young as compared to the old. The manometry evaluation was performed with the 36-sensor high-resolution solid-state manometry catheter (Sierra Scientific Instruments Inc., Los Angeles, CA, USA). Propofol inflated the LES pressure in young, approximately $10-20 \%$. The presentation of the post-deglutitive relaxation of the LES would have been really interesting. The IRP describes the deglutitive relaxation of the LES [15]. Hence exploring the impact of propofol on IRP would be the most important step in determining the reliability of HRM conducted with sedation assistance. To the best of our knowledge, there have been no clear published data or consensus studying the impact of the propofol on IRP. However, some experts do opine about possible inflation in IRP after sedation. Thus, in the patients undergoing sedation-assisted motility, interpretation of IRP results should be done in conjunction with the esophageal motility pattern and the clinical parameters. Team involved in care of such patients should be mindful about propofol effects before making a motility diagnosis. Extra-caution should be implemented for making a diagnosis in patients with elevated IRP with normal esophageal motility.

Fung et al first described the use of the midazolam in the pediatric population to assist with the esophageal manometry [10]. The study describes the safety and the hemodynamic monitoring in the infants undergoing esophageal manometry. The LES pressure was measured but the impact of the sedation on the esophageal manometry was not described. Marsh et al described the effect of midazolam on esophageal motility on human volunteers [16]. Their study results depict increase in LES residual pressure and decrease in percentage relaxation. The residual pressure is now better defined as IRP [17]. The sedation-induced inflation in IRP can certainly lead to misinterpretation and misclassification of the esophageal motility pattern. Marsh et al also demonstrated a new non-specific motility pattern in the presence of midazolam. In view of present literature, use of midazolam for the sedation assistance prior to manometry should be avoided.

The opioid administration has been known to cause wide range of the esophageal abnormalities [18]. It can alter the LES relaxation and simulate the achalasia motility pattern. Opioids administered within $24 \mathrm{~h}$ of manometry have not only shown to alter the IRP, but also affect the distal latency [19]. However, the optimal duration of opioid free period interval prior to the esophageal manometry is not defined [19]. All attempts should be made to refrain from opioid use while performing endoscopy for esophageal motility evaluation [20].

Despite our protocol for the sedation-assisted HRM, three patients received midazolam and fentanyl during the recovery phase of the endoscopy. The post-sedation agitation does pose a risk of the accidental withdrawal of the manometry catheter. If the verbal counselling is ineffective, then anxiolytics or the analgesics are used. If the administration of benzodiazepines or opioids is unavoidable, then use of such medications should certainly be accounted towards motility interpretation. The esophageal motility was normal in three study cases that received midazolam and fentanyl and posed no interference with the management.

There is a diagnostic concern in sedation-assisted HRM for patients with the isolated finding of the elevated IRP. Beyond achalasia, the patients with elevated IRP with normal esophageal peristalsis are classified as EGJ outflow obstruction [21]. Atypical achalasia can manifest as EGJ obstruction; however, it will require further evaluation with imaging studies and endoscopic ultrasound prior to concluding the diagnosis as atypical achalasia [22]. Role of sedation in IRP inflation though not clear, can alter the diagnosis from normal esophageal motility to EGJ obstruction. The likelihood of atypical achalasia cannot be ruled out. In presence of the atypical symptoms, there is a dilemma in the further management of patients. In our study, one patient (case 9) had IRP of 20 on the study acquired by the $\mathrm{Zvu}^{\circledR}$ - diagnostic software (Diversatek Healthcare) and no peristaltic abnormality was noted; on applying the possible $10 \%$ 
correction, the corrected IRP will decrease to 18 and would deem the diagnosis to be normal esophageal motility. In that particular patient, motility study was performed for evaluation of dysphagia. Endoscopic ultrasound can be offered to rule out other etiology like EGJ obstruction, but our patient refused the further procedure and hence observant management was pursued.

In our study, we were not able to perform the esophageal manometry in around $7 \%$ of the patients. There was no alternative measure to evaluate the motility, and the gold standard remains HRM. The manometry catheter has to traverse the EGJ to evaluate the LES. HRM has been also recommended for the evaluation of patients considering anti-reflux or hiatal hernia surgery. The pre-operative IRP evaluation to rule out the achalasia is the standard of care [23]. In high volume centers as well, the manometry catheter could not traverse EGJ in $29 \%$ of the cases, hence increasing the pool of patients who will remain uninvestigated without an alternative approach. Hence, while awaiting the future modalities for evaluating the lower esophageal obstruction, sedation or endoscopy assistance remains the only viable option to perform appropriate pre-operative assessment.

The imaging studies done prior to manometry should be thoroughly reviewed to evaluate for any suspicious findings of achalasia. HRM is the gold standard test for diagnosing achalasia. The imaging studies have low sensitivity for the diagnosis of achalasia [24], the data on the specificity are uncertain but they do support the diagnosis of achalasia [25]. The clinical acumen along with symptoms should be exploited to arrive to a conclusion in a patient with sedationassisted HRM. In our study, one patient with achalasia did not have the barium esophagogram; in others it certainly corroborated the findings of achalasia. It assisted in the decision making to implement the future surgical or the endoscopic intervention.

In patient with mega-esophagus one should be cautious for the sedation-related aspiration [26]. It can develop in $10 \%$ of patients with achalasia [27] and esophagectomy is the mainstay of treatment. The esophageal retention of the prior ingested food increases risk of sedation-induced aspiration. Also, the saline swallow for HRM poses a risk for aspiration.

\section{Conclusion}

The esophageal manometry should be performed in awake state. The shortcomings like patient intolerance or difficulty in negotiating the manometry catheter across the EGJ may necessitate use of sedation or the endoscopy assistance. Though not clearly proven, one should account for minimal effects of the intravenous sedation on key manometry findings as interpreted by CC. Extra-caution should be imparted in patients with isolated IRP elevation with normal esophageal motility. The corroborative findings of the barium esophagogram can be used to arrive to a clinical diagnosis. More studies are needed to explore an alternative approach or validate currently available correction used for sedation-assisted esophageal manometry parameters for patients intolerant to awake HRM.

\section{Acknowledgments}

None.

\section{Financial Disclosure}

None.

\section{Conflict of Interest}

All authors declare no conflict of interest.

\section{Informed Consent}

All participants have given written informed consent before inclusion in the study.

\section{Author Contributions}

HT: concept and design, analysis and interpretation of data; drafting of the manuscript; critical revision of the manuscript for important intellectual content. JM: concept and design, analysis and interpretation of data; drafting of the manuscript; critical revision of the manuscript for important intellectual content; study supervision. CC: acquisition of data. UMK: analysis and interpretation of data; drafting of the manuscript; revision of the manuscript. AR: acquisition of data. HP: concept and design, analysis and interpretation of data, critical revision of the manuscript for important intellectual content; study supervision.

\section{References}

1. Kahrilas PJ, Bredenoord AJ, Fox M, Gyawali CP, Roman $\mathrm{S}$, Smout AJ, Pandolfino JE, et al. The Chicago Classification of esophageal motility disorders, v3.0. Neurogastroenterol Motil. 2015;27(2):160-174.

2. Pandolfino JE, Roman S. High-resolution manometry: an atlas of esophageal motility disorders and findings of GERD using esophageal pressure topography. Thorac Surg Clin. 2011;21(4):465-475.

3. Bredenoord AJ, Fox M, Kahrilas PJ, Pandolfino JE, Schwizer W, Smout AJ, International High Resolution Manometry Working G. Chicago classification criteria of esophageal motility disorders defined in high resolution esophageal pressure topography. Neurogastroenterol Motil. 2012;24(Suppl 1):57-65.

4. Patel A, Ding A, Mirza F, Gyawali CP. Optimizing the high-resolution manometry (HRM) study protocol. Neurogastroenterol Motil. 2015;27(2):300-304.

5. Roman S, Kahrilas PJ, Boris L, Bidari K, Luger D, Pandolfino JE. High-resolution manometry studies are frequently imperfect but usually still interpretable. Clin 
Gastroenterol Hepatol. 2011;9(12):1050-1055.

6. Xiao Y, Nicodeme F, Kahrilas PJ, Roman S, Lin Z, Pandolfino JE. Optimizing the swallow protocol of clinical high-resolution esophageal manometry studies. Neurogastroenterol Motil. 2012;24(10):e489-496.

7. Allen ML, Orr WC, Mellow MH, Robinson MG. Water swallows versus food ingestion as manometric tests for esophageal dysfunction. Gastroenterology. 1988;95(3):831833.

8. Brun R, Staller K, Viner S, Kuo B. Endoscopically assisted water perfusion esophageal manometry with minimal sedation: technique, indications, and implication on the clinical management. J Clin Gastroenterol. 2011;45(9):759-763.

9. Christian KE, Morris JD, Xie G. Endoscopy- and monitored anesthesia care-assisted high-resolution impedance manometry improves clinical management. Case Rep Gastrointest Med. 2018;2018:9720243.

10. Fung KP, Math MV, Ho CO, Yap KM. Midazolam as a sedative in esophageal manometry: a study of the effect on esophageal motility. J Pediatr Gastroenterol Nutr. 1992;15(1):85-88.

11. Kempf J, Heinrich H, Reusch CE, Kook PH. Evaluation of esophageal high-resolution manometry in awake and sedated dogs. Am J Vet Res. 2013;74(6):895-900.

12. de Leon A, Ahlstrand R, Thorn SE, Wattwil M. Effects of propofol on oesophageal sphincters: a study on young and elderly volunteers using high-resolution solid-state manometry. Eur J Anaesthesiol. 2011;28(4):273-278.

13. Turan A, Wo J, Kasuya Y, Govinda R, Akca O, Dalton JE, Sessler DI, et al. Effects of dexmedetomidine and propofol on lower esophageal sphincter and gastroesophageal pressure gradient in healthy volunteers. Anesthesiology. 2010;112(1):19-24.

14. Mittal RK, Rochester DF, McCallum RW. Electrical and mechanical activity in the human lower esophageal sphincter during diaphragmatic contraction. J Clin Invest. 1988;81(4):1182-1189.

15. Carlson DA, Pandolfino JE. High-resolution manometry in clinical practice. Gastroenterol Hepatol (N Y). 2015;11(6):374-384.

16. Marsh JK, Hoffman SM, Dmuchowski CF. Effect of intravenous midazolam on esophageal motility testing in normal human volunteers. Am J Gastroenterol. 1993;88(6):860-863.

17. Conklin JL. Evaluation of esophageal motor function with high-resolution manometry. J Neurogastroenterol Motil. 2013;19(3):281-294.

18. Kraichely RE, Arora AS, Murray JA. Opiate-induced oesophageal dysmotility. Aliment Pharmacol Ther. 2010;31(5):601-606.

19. Ratuapli SK, Crowell MD, DiBaise JK, Vela MF, Ramirez FC, Burdick GE, Lacy BE, et al. Opioid-Induced Esophageal Dysfunction (OIED) in patients on chronic opioids. Am J Gastroenterol. 2015;110(7):979-984.

20. Kahrilas PJ, Bredenoord AJ, Carlson DA, Pandolfino JE. Advances in Management of Esophageal Motility Disorders. Clin Gastroenterol Hepatol. 2018;16(11):16921700.

21. Schlottmann F, Patti MG. Primary Esophageal Motility Disorders: Beyond Achalasia. Int J Mol Sci. 2017;18(7):1399.

22. Roman S, Kahrilas PJ. Challenges in the swallowing mechanism: nonobstructive dysphagia in the era of highresolution manometry and impedance. Gastroenterol Clin North Am. 2011;40(4):823-835, ix-x.

23. Jobe BA, Richter JE, Hoppo T, Peters JH, Bell R, Dengler WC, DeVault K, et al. Preoperative diagnostic workup before antireflux surgery: an evidence and experiencebased consensus of the Esophageal Diagnostic Advisory Panel. J Am Coll Surg. 2013;217(4):586-597.

24. El-Takli I, O'Brien P, Paterson WG. Clinical diagnosis of achalasia: how reliable is the barium x-ray? Can J Gastroenterol. 2006;20(5):335-337.

25. Pohl D, Tutuian R. Achalasia: an overview of diagnosis and treatment. J Gastrointestin Liver Dis. 2007;16(3):297303.

26. Bazerbachi F, Blackmon SH, Ravi K, Wong Kee Song LM. Endoscopic esophagoplasty for megaesophagus with sump stasis in end-stage achalasia. VideoGIE. 2017;2(11):274-275.

27. Orringer MB, Stirling MC. Esophageal resection for achalasia: indications and results. Ann Thorac Surg. 1989;47(3):340-345. 